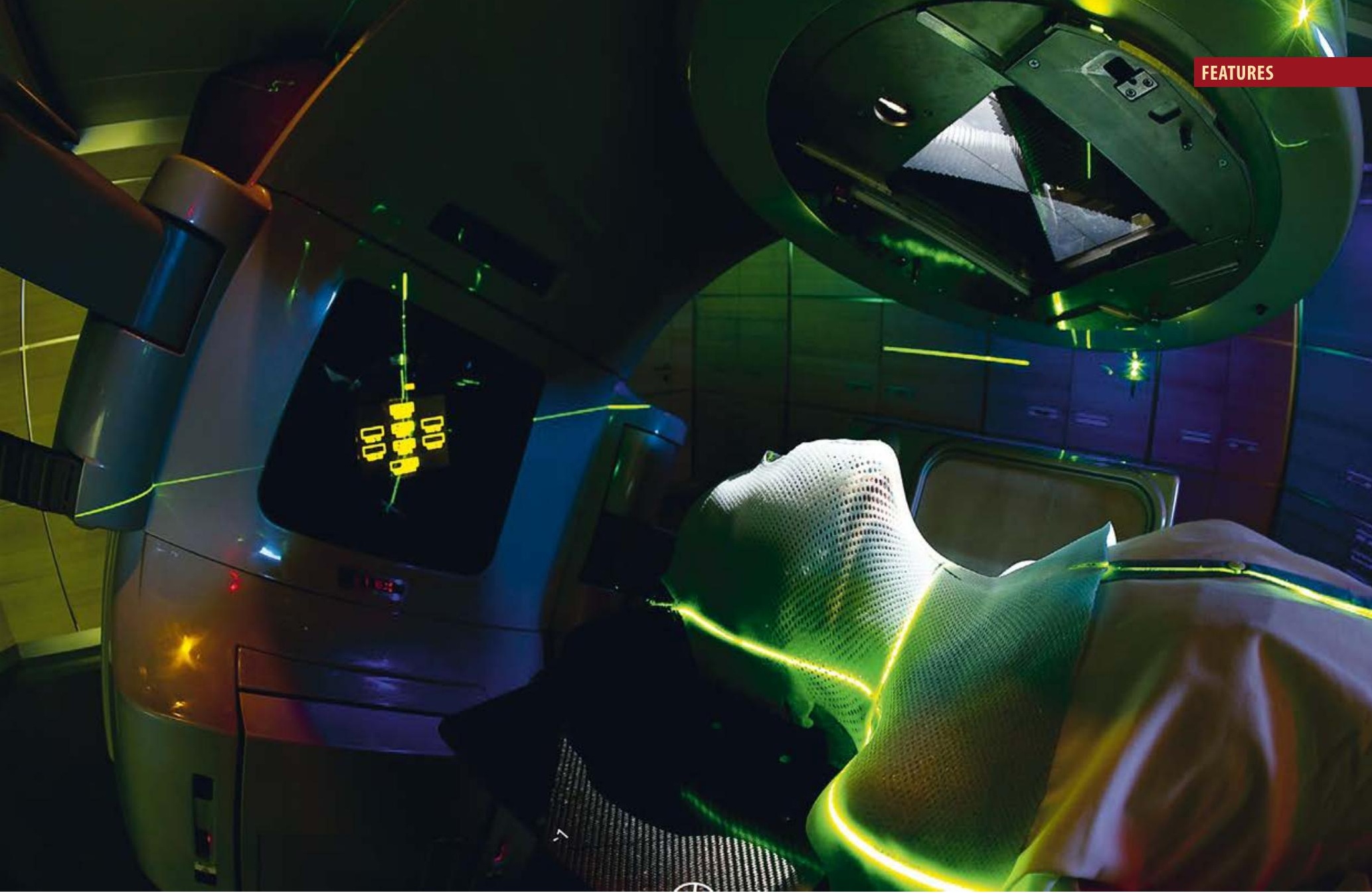

\title{
FROM PARTICLE PHYSICS TO MEDTECH AND BIOMEDICAL RESEARCH
}

\author{
- Manuela Cirilli, CERN - Dol: https://doi.org/10.1051/epn/2018507
}

Physics phenomena underpin many techniques and technologies that are used for both diagnosis and treatment of a variety of diseases. This is the case for radiotherapy, Magnetic Resonance Imaging (MRI), and Positron Emission Tomography (PET) that are based on our knowledge, respectively, of how particles interact with matter, of how atomic nuclei behave in oscillating magnetic fields, and of how positron decay.

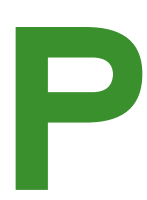

article physics has also played a role in developing innovative technologies that made their way into the Medical Technology (MedTech) sector: frontier instruments like the Tevatron at Fermilab or the Large Hadron Collider (LHC) at CERN, and their detectors, require frontier technologies, well beyond the industrial know-how at the time these experiments were conceived. Advances in fields including accelerators, detectors, and computing have had a positive impact in many fields of society, and in particular in medical and biomedical technologies and research.

\section{Accelerator technologies for health}

Linear accelerators are routinely used in hospitals for conventional cancer radiotherapy with X-rays. Radiotherapy is a mainstay of cancer treatment, its main limitation being the maximum tolerated dose to healthy tissues traversed by the X-ray beam: in fact, photons loose energy slowly and mainly exponentially as they penetrate tissue, meaning that they will deposit radiation dose before and after the target tumour. Nowadays, techniques such as intensity-modulated radiation therapy (IMRT), image-guided radiation therapy (IGRT), $\triangle$ One of the most important of all applications of particle accelerators is their application in the treatment of cancer. Photo credits: CERN 


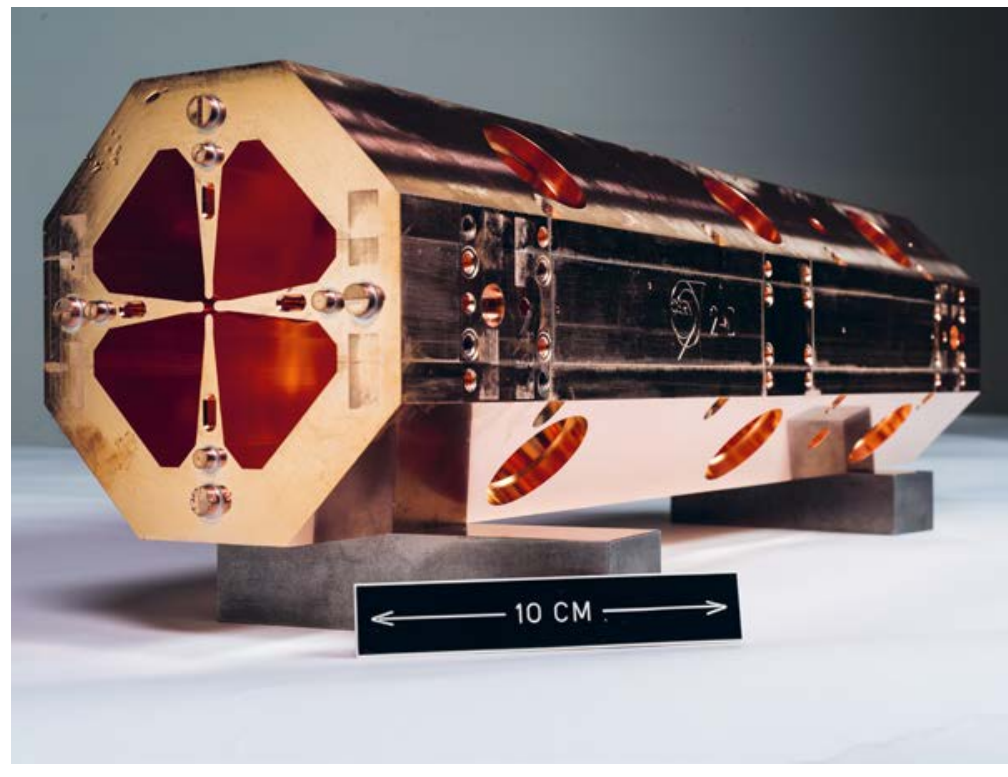

\FIG. 1: An innovative linear accelerator designed by CERN

to be compact, modular, low-cost

and suitable for medical applications. Photo credits: CERN

and stereotactic radiotherapy (SBRT) enable precise delivery of the dose to the exact volume of the tumour while minimising the irradiation of healthy tissues.

A novel form of radiation therapy was proposed in 1946 by accelerator pioneer Robert R. Wilson, first Director of Fermilab, who published an article in Radiology about the therapeutic interest of proton beams for treating cancer. The clinical interest in hadron therapy resides in the fact that it delivers precision treatment of tumours, exploiting the characteristic shape of the dose deposition as a function of the depth of matter traversed: unlike photons, hadrons deposit almost all of their energy in a sharp peak - the Bragg peak - at the very end of their path.

The Bragg peak makes it possible to target a well-defined cancerous region at a depth in the body that can be tuned by adjusting the energy of the incident particle beam, with reduced damage to the surrounding healthy tissues. The dose deposition is so sharp that new techniques had to be developed to treat the whole target. These fall under the categories of passive scattering, where one or more scatterers are used to spread the beam, and spot scanning, where a thin, pencil-like beam covers the target volume in $3 \mathrm{D}$ under the control of sweeping magnets coupled to energy variations.

While the advantages of protons over photons are quantitative in terms of the amount and distribution of the delivered dose, several studies show evidence that carbon ions damage cancer cells in a way that they cannot repair themselves. Carbon therapy may be the optimal choice to tackle radio-resistant tumours; other ions, such as helium, are also being investigated ${ }^{2}$.

\footnotetext{
${ }^{1}$ https://cerncourier.com/the-changing-landscape-of-cancer-therapy/

${ }^{2}$ http://cds.cern.ch/record/1734611/files/vol51-issue10-p037-e.pdf

${ }^{3}$ https://www.symmetrymagazine.org/article/december-2008/deconstruction-mri
}

The complexity of the infrastructures required for hadron therapy is a limiting factor for its exploitation, and particle physics has constantly contributed to advancing the needed accelerator technologies (Figure 1). The first patients were indeed being treated in physics research institutes, starting in Berkeley, until the construction of the first dedicated proton therapy centre in Loma Linda (California). While various companies are now offering turn-key solutions for proton therapy centres and even single-room systems, custom design - often realised in partnership with research institutions - is still the norm for multi-ion facilities.

Accelerators are also increasingly needed for the production of radioisotopes, which are used in nuclear medicine imaging and therapy. Artificially-made radioisotopes are still mostly produced by research reactors, which are frequently shut down for maintenance. Dedicated cyclotrons are playing an increasingly relevant role, and new accelerator-based technologies are being explored for next-generation facilities that would replace the wobbly reactors. The ISOLDE accelerator facility at CERN has constantly been developing the so-called Isotope Separation Online (ISOL) technique, which is now being taken one step further by the CERN-MEDICIS installation, dedicated to the production of a wide range of innovative radioisotopes for medical and biomedical research.

The growing energy reach of particle accelerators has pushed the development of novel superconducting magnet technologies able to generate higher magnetic fields: this is the case of Niobium-titanium (Nb-Ti), an alloy that was identified in 1962 as having superior superconducting properties paired with easy workability. In the early seventies, just when Fermilab started building the Tevatron accelerator, American chemist Paul Lauterbur published on Nature his paper describing a new imaging technique based on NMR. Both the Tevatron design and the infant MRI technology were in dire need of very strong magnetic fields, which could only be provided by $\mathrm{Nb}$-Ti coils that nobody was capable of producing at that time with the necessary specifications. This is where the role of big science in pushing technologies beyond stateof-the-art becomes manifest: Fermilab bought the raw material in quantities that were orders of magnitude larger than standard orders for $\mathrm{Nb}$-Ti at that time, and worked alongside manufacturers to achieve the perfect wires for the Tevatron. This paved the way for commercial use of Nb-Ti in MRI machines and, later-on, in medical accelerators ${ }^{3}$.

\section{Particle detectors}

Medical imaging has radically transformed medicine, changing the way doctors can detect, diagnose, and treat a variety of diseases. Technological breakthroughs have made imaging faster, more precise, and less invasive, generating a wealth of new imaging 
techniques and methods. These innovations have often been driven by the latest developments in particle detectors and electronics, whose increasingly challenging requirements keep pushing technologies beyond state-of-the-art.

The epitome of the cross-fertilization between particle physics detectors and imaging tools is the technology for PET scanners. As in the case of Nb-Ti for MRI, high-energy physics has played a major role in making scintillating crystals available at industrial scale and affordable (Figure 2). The calorimeters of the Crystal Ball detector at the Stanford Linear Accelerator Center and of the L3 experiment at the Large Electron Positron (LEP) collider at CERN pioneered, respectively, the large-scale use of sodium iodide (NaI) and bismuth germanate (BGO), two key scintillators for PET scanners. Similarly, the mammoth scale of the CMS detector at the LHC required the development of 120000 avalanche photodiodes (APD) for scintillator readout in a high magnetic field: the CMS experience highlighted the limitations of APDs and gave a positive impulse to the $\mathrm{R} \& \mathrm{D}$ on silicon photomultipliers (SiPM), first developed by Russian institutes (Moscow and Dubna $)^{4}$.

In some cases, technologies developed for particle physics detectors have been directly transferred to the medical imaging field. A recent example is a breakthrough application of a chip developed at CERN by the Medipix3 Collaboration for LHC experiments: a New-Zealand company developed a scanner based on this technology and managed to take the first 3D colour X-ray images of a human body ${ }^{5}$ (Figure 3).

Particle detectors also find important applications in the field of dosimetry, where novel technologies can considerably improve the performance and reliability of dose monitoring procedures. Advances in radiation therapy technology - such as intensity modulated radiation therapy (IMRT), image-guided radiation therapy (IGRT) and hadron therapy - have called for an increased reliability and accuracy in dosimetric techniques. During treatment, ion chambers measure the integrated dose and shut off the beam once the required therapeutic dose, as specified by the treatment plan, has been reached. Before treatment, periodic Quality Assurance procedures are required to verify for example the homogeneity of the radiation field, by means of radiochromic films or a matrix of active monitors such as pin diodes or ion chambers. In hadron therapy, dosimetry also implies the verification of the position of the Bragg peak (where the majority of the radiation is deposited) in a water phantom. Additional in-vivo dose measurements during the actual radiation treatment of an individual patient represent an additional safety measure. The relatively recent field of micro dosimetry also needs sophisticated detectors to detect the energy deposited in cellular and sub-cellular structures.

\section{Computing and simulation}

Computer simulations are ubiquitous in medical applications, from detector modelling, to accelerator studies for medical use, shielding design, radioisotope production, treatment planning for radiation therapy, evaluation of radiation exposure for astronauts. Particle physics excels in the domain of Monte Carlo (MC) simulations, and simulation codes initially developed for High-Energy Physics (HEP), such as Geant 4 and FLUKA ${ }^{6}$, have also become crucial to modelling the effects of radiation on biological tissues for a variety of applications in the medical field.

Geant4 is true reflection of the collaborative model of HEP: it is a world-wide collaboration of scientists and software engineers whose goal is to develop, maintain and provide support for the Geant 4 toolkit. Today, it is adopted by thousands of users worldwide for application in domains beyond high-energy physics ${ }^{7}$ : examples of Geant 4 extensions for use in the medical field are GATE, TOPAS and Geant4-DNA. The latter offers the possibility to model physical, physicochemical, and chemical processes up to the microsecond scale, therefore allowing for the simulation of early biological effects induced by ionizing radiation at the subcellular scale.

Among other medical applications, Geant4 is being used to study the radiation environment on the ISS, as well as radiation effects on possible future manned space missions to the Moon or Mars.
V FIG. 2: Scintillating crystals are used both in particle detectors for high-energy physics experiments and in PET scanners to detect photons. The photo shows some of the almost 80000 precision-grown crystals for the CMS experiment at CERN. Photo credits: Peter Ginter/CERN

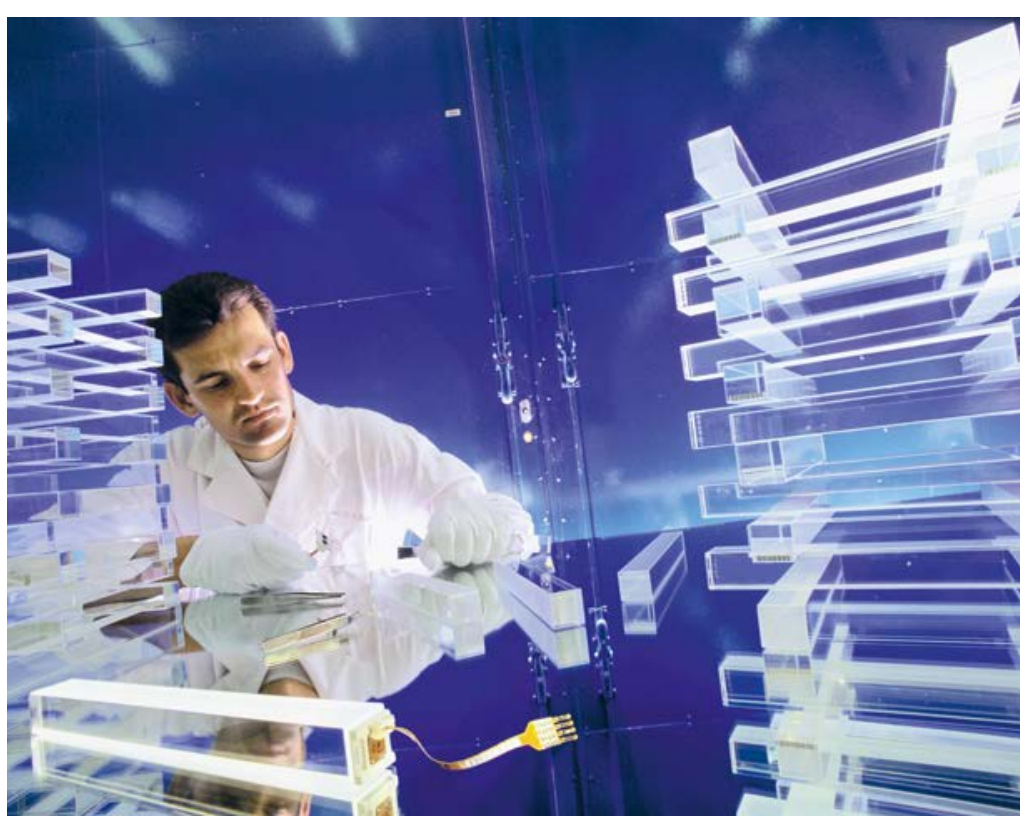

${ }^{4}$ https://indico.cern.ch/event/164917/contributions/1417121/attachments/ 198512/278663/PhotoDet12_-_collazuol_-_v3.pdf

${ }^{5}$ https://home.cern/about/updates/2018/07/first-3d-colour-x-ray-humanusing-cern-technology

${ }^{6}$ http://www.fluka.org/fluka.phpw

http://www.geant4.org/geant4/applications.1 


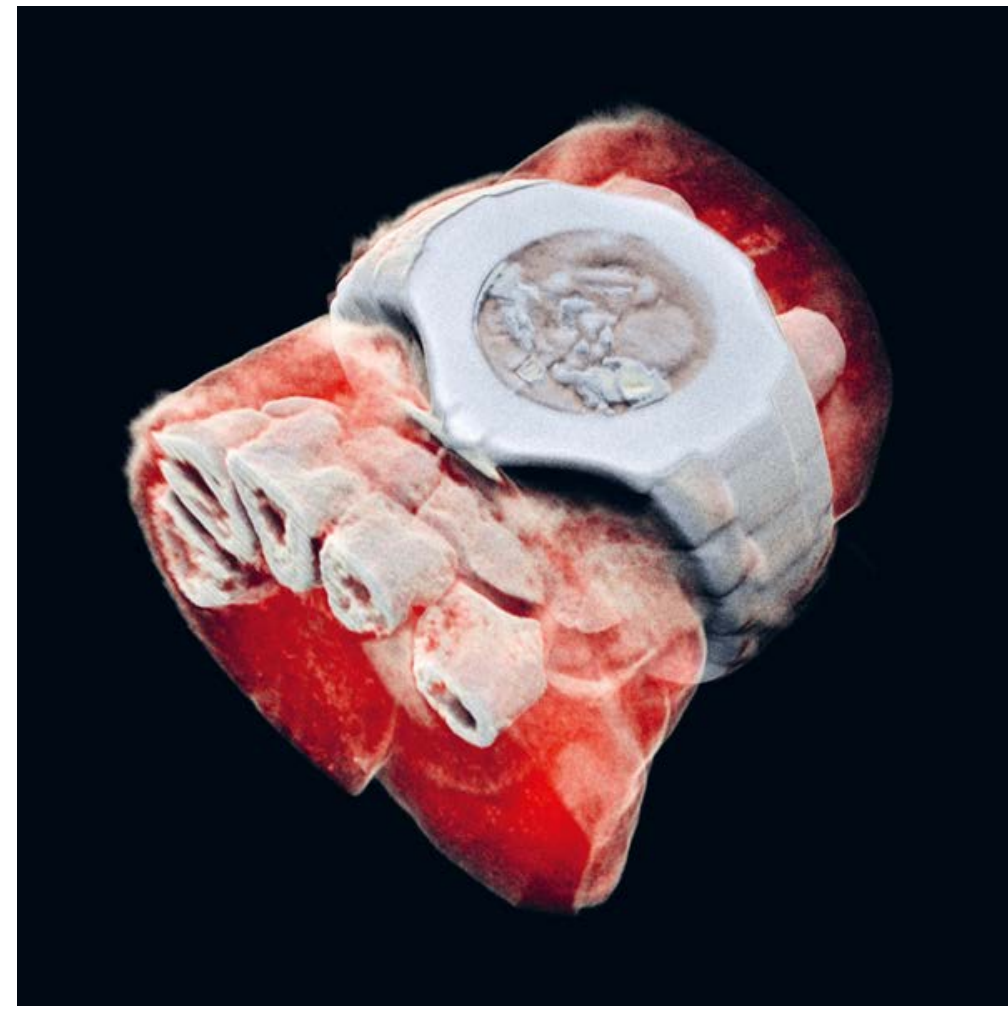

$\triangle$ FIG. 3: A 3D image of a wrist with a watch showing part of the finger

bones in white and soft tissue in red, taken with the MARS scanner using the technology developed by the Medipix3 collaboration at CERN. Photo credits: MARS Bioimaging Ltd.
Computing tools, infrastructures and services developed for HEP have also great potential for applications in the medical and biomedical field. Health is a vital part of society, and as such is affected by the same technology trends, including the pervasive use of computer techniques for data sharing and patient referral or the rise of Artificial Intelligence (AI) and Machine Learning (ML) techniques. The "big data" trend in the healthcare sector is going strong, as doctors and researchers realise that they are sitting on a goldmine of data. Medical data comes from many different types of sources (instruments, sensors, imaging devices, environmental data), disciplines (genome sequencing, metabolomics, metagenomics) and formats (publications, digital data sets, software code). It is therefore increasingly difficult for researchers to make efficient use of the available inputs, analyse data, store and share results. Furthermore, most of the medical data is produced by a large number of institutes and has stringent privacy and confidentiality constraints. The increasingly large quantity and variety of data make the discovery, identification, reuse and reproducibility of relevant data sets and results more and more difficult. Data analytics tools hold the promise of better disease identification and diagnosis, improved efficiency e.g. in screening new pharmaceuticals, increasingly personalised treatments, better outbreak prediction or novel intelligent electronic health records. Meanwhile, the HEP community has been applying ML techniques for quite some time, and in particular since the LHC started data-taking in 2010 generating an unprecedented amount of collisions and of data from the equipment. The challenges faced in HEP are similar to those in other fields, including the medical sector: they range from speeding up computer simulations, developing modern code optimisation techniques, to real-time analysis, particle reconstruction and identification, monitoring of detectors, hardware anomalies and preemptive maintenance.

\section{Conclusions}

Innovative ideas and technologies from particle physics have been playing an increasingly important role in healthcare over the past 100 years, since the advent of radiation-based medical diagnosis and treatment following the discovery of X-rays and radioactivity. Nowadays, state-of-the-art techniques derived from particle accelerators, detectors, and physics computing are routinely used in clinical practice and medical and biomedical research: from technology for PET scanners, to dedicated accelerators for cancer therapy, to simulations and data analytics instruments.

As physicists continue to develop cutting-edge instrumentation to achieve their far-reaching goals, the MedTech and biomedical sector will be able to reap the benefits of the technological advances. Examples are: novel accelerator designs that would allow more effective and affordable ion therapy for cancer treatment; research and development aimed at improving the time resolution of scintillating crystals, which could lead to dramatic improvements in the sensitivity of PET scanners; more powerful ML tools driven by the exacting needs of particle physics, which could be applied to similar challenges in the medical field.

Last but not least, the experience gained in the organisation of particle physics projects can contribute to the shaping of medical research: HEP collaborations have been bringing together thousands of scientists from every corner of the world, and particle physicists have learned to work collectively for a common goal, to rely on consensus to take decisions, and to implement flexible yet effective management structures for their experiments. This collaborative framework can serve as model and inspiration for medical and biomedical research, where multidisciplinary, multinational collaboration is beginning to bloom.

\section{About the Author}

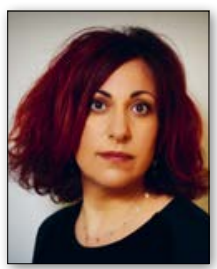

Manuela Cirilli works at CERN, the European Organization for Nuclear Research, where she leads the Medical Applications section of CERN's Knowledge Transfer group. Prior to this, she was an experimental researcher in particle physics: first in the NA48 experiment at CERN, aimed at measuring $\mathrm{CP}$ violation in the kaon system, and then in the ATLAS collaboration at CERN's Large Hadron Collider. In parallel to her scientific career, Manuela has been engaging in science communication and popularization, and in promoting STEM careers among young women and girls. 